\title{
Etiological Analysis of 419 Cases of Digestive Tract Hemorrhage
}

\author{
Shibao Song ${ }^{*}$, Dong Wang ${ }^{*}$, Chuanxin Zou ${ }^{2 \#}$ \\ ${ }^{1}$ The Second Clinical Medical College, Yangtze University, Jingzhou, China \\ ${ }^{2}$ Department of Gastroenterology, Jingzhou Central Hospital, The Second Clinical Medical College, Yangtze University, \\ Jingzhou, China \\ Email: "1559734687@qq.com
}

How to cite this paper: Song, S.B., Wang, D. and Zou, C.X. (2018) Etiological Analysis of 419 Cases of Digestive Tract Hemorrhage. Yangtze Medicine, 2, 178-185. https://doi.org/10.4236/ym.2018.23019

Received: July 27, 2018

Accepted: August 27, 2018

Published: August 30, 2018

Copyright $\odot 2018$ by authors and Scientific Research Publishing Inc. This work is licensed under the Creative Commons Attribution International License (CC BY 4.0).

http://creativecommons.org/licenses/by/4.0/

\begin{abstract}
Aim: To study common cause of digestive tract hemorrhage and the relationship between etiology and age. Methods: Retrospective analysis about the data of 419 cases of digestive tract hemorrhage in digestive internal medicine from January 2016 to January 2018 in Jingzhou Central Hospital. Results: 419 cases of digestive tract hemorrhage include 305 cases of hemorrhage in upper digestive tract and 114 cases of hemorrhage in lower digestive tract. The first 5 causes of upper digestive tract hemorrhage in middle-aged and young group were: duodenal bulb ulcer, gastric ulcer, esophageal and stomach bottom varices bleeding in cirrhosis of schistosomiasis, compound ulcer, esophageal and stomach bottom varices bleeding in hepatitis B cirrhosis. The first 5 causes of upper digestive tract hemorrhage in elderly group were: gastric ulcer, duodenal bulb ulcer, esophageal and stomach bottom varices bleeding in hepatitis B cirrhosis, esophageal and stomach bottom varices bleeding in cirrhosis of schistosomiasis, gastric cancer. The first 5 causes of lower digestive tract hemorrhage in middle-aged and young group were: ulcerative colitis, colorectal polyps, hemorrhoids, crohn's disease, ischemic bowel disease. The first 5 causes of lower digestive tract hemorrhage in elderly group were: colorectal polyps, colon cancer, ulcerative colitis, ischemic bowel disease, hemorrhoids. Conclusion: In the cases of upper digestive tract hemorrhage, peptic ulcer should be paid attention to in middle-aged and young people, and elderly should be noted in gastric cancer. Ulcerative colitis should be paid attention to in middle-aged and young people with lower digestive tract hemorrhage, and elderly should be noted in colorectal polyps and colon cancer.
\end{abstract}

\section{Keywords}

Digestive Tract Hemorrhage, Upper Digestive Tract Hemorrhage, Lower Digestive Tract Hemorrhage, Etiology Analysis

${ }^{*}$ Contributed equally. 


\section{Introduction}

Digestive tract hemorrhage is a common clinical disease, both upper digestive tract hemorrhage and lower tract hemorrhage, which is characterized by acute onset and dangerous condition. So to judge the etiology of digestive tract hemorrhage quickly and give corresponding treatment is very important [1]. Previous academics have studied the causes of digestive tract hemorrhage, but the main research group focused on the elderly people. And the etiology analysis of lower tract hemorrhage was more reports [2] [3]. In this paper, there will be retrospective analysis about the data of 419 cases of digestive tract hemorrhage in digestive internal medicine from January 2016 to January 2018 in Jingzhou Central Hospital. The causes of upper digestive tract hemorrhage and lower digestive tract hemorrhage were analyzed in two parts, in order to provide reference for the diagnosis and treatment of digestive tract hemorrhage in clinic.

\section{Materials and Methods}

\subsection{Case Data}

A retrospective analysis of 419 cases of digestive tract hemorrhage that have complete clinical data and confirmed in Jingzhou Central Hospital digestive internal medicine from January 2016 to January 2018. In this study, age is the variable, and the subjects were divided into the middle-aged and young group as well as elderly group. The minimum age for the case was 15 years old, and the maximum age is 85 years old. Younger than 60 years old are young and middle-aged groups, and the remaining cases are the elderly group. Among them, 305 cases of upper digestive tract hemorrhage, 227 cases of male, 78 cases of female, 178 cases of middle-aged and young group (15 - 59 years old), 127 cases of elderly group (60 - 85 years old). 114 cases of lower digestive tract hemorrhage, 64 cases of male, 50 cases of female, 80 cases of middle-aged and young group (15 - 59 years old), 34 cases of elderly group (60 - 85 years old).

\subsection{Inclusion Criteria}

All retrospective study patients were diagnosed with electronic gastroscopy or electronic colonoscopy and other clinical criteria (the clinical symptoms of hematemesis, black feces, or the fecal occult blood test are positive, bleeding spots or active bleeding under electronic gastroscope or electronic colonoscopy). An electronic gastroscope or electronic colonoscopy operator has more than 5 years of experience in digestive endoscopy.

\subsection{Statistical Analysis}

SPSS23.0 statistical software was used for statistical analysis. Comparison between young group and elderly group using $\chi^{2}$ test or Fisher exact probability method of the four-grid table data, $\mathrm{P}<0.05$ was considered statistically significant. 


\section{Results}

Of all the selected clinical cases, there were 291 males and 128 females. Among men, 153 cases were hematemesis or black stool, 138 cases were positive for fecal occult blood test. Among women, 75 cases were hematemesis or black stool, 53 cases were positive for fecal occult blood test. The specific results are shown in Table 1.

\subsection{Etiology Composition of Upper Digestive Tract Hemorrhage}

305 cases of upper digestive tract hemorrhage include 178 cases of middle-aged and young group, 127 cases of elderly group. The distribution of main etiology in the young and middle-aged group was as follows: 130 cases of ulcerative disease (73.03\%), which include 74 cases of duodenal bulb ulcer (41.57\%), 35 cases of gastric ulcer (19.66\%), 13 cases of compound ulcer (7.30\%), 4 cases of residual gastric ulcer (2.25\%), 3 cases of anastomotic ulcer (1.69\%), 1 case of esophageal ulcer (0.56\%). 36 cases of cirrhosis of the esophagus and gastric fundus varicose hemorrhage (20.22\%), which include 24 cases of esophageal and stomach bottom varices bleeding in cirrhosis of schistosomiasis (13.48\%), 10 cases of esophageal and stomach bottom varices bleeding in hepatitis B cirrhosis (5.62\%), 2 cases of esophageal and stomach bottom varices bleeding in alcoholic cirrhosis $(1.12 \%)$. The distribution of main etiology in the elderly group was as follows: 78 cases of ulcerative disease (61.42\%), which include 35 cases of gastric ulcer (27.56\%), 24 cases of duodenal bulb ulcer (18.90\%), 7 cases of compound ulcer (5.51\%), 6 cases of residual gastric ulcer $(4.72 \%), 4$ cases of esophageal ulcer (3.15\%), 2 cases of anastomotic ulcer (1.57\%). 28 cases of cirrhosis of the esophagus and gastric fundus varicose hemorrhage (22.05\%), which include 13 cases of esophageal and stomach bottom varices bleeding in hepatitis B cirrhosis (10.24\%), 12 cases of esophageal and stomach bottom varices bleeding in cirrhosis of schistosomiasis (9.45\%), 3 cases of esophageal and stomach bottom varices bleeding in alcoholic cirrhosis (2.36\%). 13 cases of malignant tumor (10.24\%), which include 11 cases of gastric cancer (8.66\%), 2 cases of esophageal cancer (1.57\%). The statistical results show that duodenal bulb ulcer is predilection in the young and middle-aged group, and gastric cancer is predilection in the elderly group. The differences have statistically significant. Although the incidence of other diseases was different, the difference was not statistically significant. Statistical analysis of the etiology between the two groups is shown in Table 2.

\subsection{Etiology Composition of Lower Digestive Tract Hemorrhage}

114 cases of lower digestive tract hemorrhage, 80 cases of middle-aged and

Table 1. Clinical characteristics of the patients.

\begin{tabular}{ccc}
\hline Clinical manifestation & Male cases (case) & Female cases (case) \\
\hline Hematemesis or black stool & 153 & 75 \\
Fecal occult blood test positive & 138 & 53 \\
\hline
\end{tabular}


Table 2. The distribution of upper digestive tract hemorrhage in all age groups case (\%).

\begin{tabular}{|c|c|c|c|}
\hline Etiology & $\begin{array}{c}\text { Young and } \\
\text { middle-aged group }\end{array}$ & $\begin{array}{l}\text { Elderly } \\
\text { group }\end{array}$ & $\mathrm{P}$ value \\
\hline duodenal bulb ulcer & $74(41.57)$ & $24(18.90)$ & $<0.01$ \\
\hline gastric ulcer & $35(19.66)$ & $35(27.56)$ & $>0.05$ \\
\hline compound ulcer & $13(7.30)$ & $7(5.51)$ & $>0.05$ \\
\hline residual gastric ulcer & $4(2.25)$ & $6(4.72)$ & $>0.05$ \\
\hline anastomotic ulcer & $3(1.69)$ & $2(1.57)$ & $>0.05$ \\
\hline esophageal ulcer & $1(0.56)$ & $4(3.15)$ & $>0.05$ \\
\hline $\begin{array}{l}\text { esophageal and stomach bottom varices } \\
\text { bleeding in cirrhosis of schistosomiasis }\end{array}$ & $24(13.48)$ & $12(9.45)$ & $>0.05$ \\
\hline $\begin{array}{l}\text { esophageal and stomach bottom varices } \\
\text { bleeding in hepatitis B cirrhosis }\end{array}$ & $10(5.62)$ & $13(10.24)$ & $>0.05$ \\
\hline $\begin{array}{l}\text { esophageal and stomach bottom varices } \\
\text { bleeding in alcoholic cirrhosis }\end{array}$ & $2(1.12)$ & $3(2.36)$ & $>0.05$ \\
\hline esophageal cancer & $0(0.00)$ & $2(1.57)$ & $>0.05$ \\
\hline liver cancer & $2(1.12)$ & $0(0.00)$ & $>0.05$ \\
\hline gastric cancer & $2(1.12)$ & $11(8.66)$ & $<0.01$ \\
\hline esophagus and cardia mucosa tear syndrome & $4(2.25)$ & $2(1.57)$ & $>0.05$ \\
\hline gastric polyps & $2(1.12)$ & $1(0.79)$ & $>0.05$ \\
\hline acute erosive gastritis & $1(0.56)$ & $4(3.15)$ & $>0.05$ \\
\hline esophageal papilloma & $1(0.56)$ & $0(0.00)$ & $>0.05$ \\
\hline chronic atrophic gastritis with erosion & $0(0.00)$ & $1(0.79)$ & $>0.05$ \\
\hline
\end{tabular}

young group, 34 cases of elderly group. The distribution of etiology in the young and middle-aged group was as follows: 49 cases of inflammatory bowel disease (61.25\%), which include 43 cases of ulcerative colitis (53.75\%), 6 cases of crohn's disease (7.50\%). 13 cases of colorectal polyps (16.25\%). 7 cases of hemorrhoids (8.75\%). 6 cases of ischemic bowel disease (7.50\%). 4 cases of colon ulcers (5.00\%). 1 case of type B paratyphoid (1.25\%). The distribution of etiology in the elderly group was as follows: 12 cases of colorectal polyps (35.29\%). 6 cases of colon cancer (17.65\%). 6 cases of inflammatory bowel disease (17.65\%), which include 5 cases of ulcerative colitis (14.71\%), 1 case of crohn's disease (2.94\%). 4 cases of ischemic bowel disease (11.76\%). 3 cases of hemorrhoids (8.82\%). 2 cases of ileocecal vascular malformation (5.88\%). 1 case of colon ulcers $(2.94 \%)$. The statistical results show that ulcerative colitis is predilection in the young and middle-aged group, colorectal polyps and colon cancer is predilection in the elderly group. The differences have statistically significant. There was no statistically significant in the incidence of other diseases. Statistical analysis of the etiology between the two groups is shown in Table 3. 
Table 3. The distribution of lower digestive tract hemorrhage in all age groups case (\%).

\begin{tabular}{cccc}
\hline Etiology & Young and middle-aged group & Elderly group & P-value \\
\hline ulcerative colitis & $43(53.75)$ & $5(14.71)$ & $<0.01$ \\
crohn's disease & $6(7.50)$ & $1(2.94)$ & $>0.05$ \\
colorectal polyps & $13(16.25)$ & $12(35.29)$ & $<0.05$ \\
hemorrhoids & $7(8.75)$ & $3(8.82)$ & $>0.05$ \\
ischemic bowel disease & $6(7.50)$ & $4(11.76)$ & $>0.05$ \\
colon ulcers & $4(5.00)$ & $1(2.94)$ & $>0.05$ \\
colon cancer & $0(0.00)$ & $6(17.65)$ & $<0.01$ \\
type B paratyphoid & $1(1.25)$ & $0(0.00)$ & $>0.05$ \\
ileocecal vascular malformation & $0(0.00)$ & $2(5.88)$ & $>0.05$ \\
\hline
\end{tabular}

\section{Discussion}

Digestive tract hemorrhage has the characteristic of acute onset and rapid progression of the course, often due to excessive blood loss leading to shock. It is a common clinical critical illness. In recent years, with the continuous development of digestive endoscope technology, especially the development of capsule endoscopy and small enteroscopy, the new concept of digestive tract segmentation has gained more and more recognition. The new definition uses the duodenal papilla and the ileocecal valve as an anatomical sign, the digestive tract is divided into the upper digestive tract, the middle digestive tract and the lower digestive tract. Thus, the small intestinal bleeding is independent, called middle digestive tract hemorrhage. The upper and lower digestive tract hemorrhage discussed in this paper is referred to in the new classification method.

In this study, it includes 305 cases of upper digestive tract hemorrhage and 114 cases of lower digestive tract hemorrhage; the ratio is $2.68: 1$; there are more cases of upper digestive tract hemorrhage than lower digestive tract hemorrhage. It is consistent with previous research results [4]. In the cases of upper digestive tract hemorrhage, it includes 227 cases of male, 78 cases of female; the proportion of males and females is 2.91:1. The number of male cases is higher than female cases. It suggests that the incidence of upper digestive tract hemorrhage is related to sex, this result is consistent with previous research in China [5]. Among them, the first 5 causes in middle-aged and young group were: duodenal bulb ulcer, gastric ulcer, esophageal and stomach bottom varices bleeding in cirrhosis of schistosomiasis, compound ulcer, esophageal and stomach bottom varices bleeding in hepatitis B cirrhosis. Of these, there are 3 types of peptic ulcer and 2 types of cirrhosis of the esophagus and gastric fundus varicose hemorrhage. In the case of duodenal bulb ulcer, the difference between young group and old group is statistically significant. This is consistent with the relevant reports [5]. This may be related to the stressful life of young people in today's society and unhealthy habits such as drinking, smoking, staying up late. In all of the causes, esophageal and stomach bottom varices bleeding in cirrhosis of 
schistosomiasis in the middle-aged and young group accounted for up to $13.48 \%$. This may be related to the location of Jingzhou in the schistosomiasis endemic area. Many people have been in contact with the epidemic water. However, there was no related examination of schistosomiasis infection until clinical symptoms appear such as esophagus and gastric fundus varicose hemorrhage. Hepatitis B cirrhosis is in the 5th place, it shows that the current status of hepatitis B infection in China is still severe [6]. Strengthening the prevention and treatment of hepatitis B is still long way to go.

In cases of upper digestive tract hemorrhage in the elderly group, the first 5 causes were: gastric ulcer, duodenal bulb ulcer, esophageal and stomach bottom varices bleeding in hepatitis B cirrhosis, esophageal and stomach bottom varices bleeding in cirrhosis of schistosomiasis, gastric cancer. Elderly patients with arteriosclerosis, gastrointestinal mucosal blood supply is not sufficient, so leading to mucosal atrophy, gastric acid and pepsin invasion could easily cause ulcers [4]. And elderly patients often have a history of taking aspirin or NSAIDS drugs. It can also cause gastric mucosal injury and gastric ulcer. The proportion of gastric cancer is $8.66 \%$, compared with the middle-aged and young groups; the difference is statistically significant. Malignant tumors are often found in elderly patients, and there are no specific manifestations in the early days. When there are obvious clinical symptoms such as hematemesis, black stool, and so on, it is in the middle and late stages. The therapeutic effect is poor. Therefore, it is of great significance to make a census of malignant tumor in elderly patients.

In the cases of lower digestive tract hemorrhage, the first 5 causes in middle-aged and young group were: ulcerative colitis, colorectal polyps, hemorrhoids, crohn's disease, ischemic bowel disease. The first 5 causes in elderly group were: colorectal polyps, colon cancer, ulcerative colitis, ischemic bowel disease, hemorrhoids. Ulcerative colitis occupation ratio in middle-aged and young group is significantly higher than in elderly group, colon cancer and colorectal polyps occupation ratio in elderly group is significantly higher than in middle-aged and young group, the difference is statistically significant, consistent with Guoxiuli and other reports [7]. Ulcerative colitis often occurs in middle and young people, clinical symptoms often occur at age 20 - 40, diarrhea and mucous blood stool are found in most patients, the prevalence of ulcerative colitis in China has increased markedly in recent years [1], this result is consistent with this study. Patients with colon cancer are still mainly occurred in elderly, it also confirms the traditional idea that malignancy is often occurs in elderly people. Colorectal polyps are the new organisms which overgrowth in the colorectal mucosa. According to the pathological results, it can be divided into inflammatory polyps, hyperplastic polyps and adenomatoid polyps, and the incidence of adenomatoid polyps is the highest. Adenomatoid polyps are divided into tubular adenoma, villous adenoma and mixed-type adenoma. At present, colon adenoma-colon cancer sequential theory has been accepted by the vast number of medical workers, the diagnosis of colonic polyps is of great significance for the prevention of colon cancer [8]. Foreign related research reports [9], the common 
cause of lower digestive tract hemorrhage are intestinal diverticulum, ischemic bowel disease, perianal disease, in this study, the common causes of lower digestive tract hemorrhage are ulcerative colitis, colorectal polyps, ischemic bowel disease, perianal disease (hemorrhoids), this difference may be related to different living habits, environmental factors and ethnic differences between China and foreign countries.

Of course, there are many shortcomings in this study. First, this study is a single center retrospective study. The number of cases is less and the etiology of digestive tract hemorrhage cannot be well represented. Secondly, the subjects included in this study were diagnosed by gastroscopy or enteroscopy. None of the patients who had symptoms of digestive tract hemorrhage without gastroscopy or enteroscopy examination were included in the study. For example, digestive tract hemorrhage is serious and surgical cases are not included in the study area. This may also affect the statistics of the digestive tract hemorrhage etiology. It is hoped that the follow-up researchers should take it as a warning, and make a better study on etiology of digestive tract hemorrhage.

\section{Conclusion}

In a word, in the etiology of upper digestive tract hemorrhage, the incidence of the duodenal ulcer in the middle-aged and young group was higher than that in the elderly group, and the incidence of the gastric cancer in the elderly group was higher than that in the middle-aged and young group. In the etiology of lower digestive tract hemorrhage, the incidence of the ulcerative colitis in the middle-aged and young group was higher than that in the elderly group, and the incidence of the colorectal polyps and colon cancer in the elderly group was higher than that in the middle-aged and young group. The difference is statistically significant. This is a reference for the digestive endoscopy physician in gastrointestinal endoscopy for patients with digestive tract hemorrhage.

\section{Acknowledgements}

We thank Yangtze University for fellowship to Shibao Song and Dong Wang as a graduate student.

\section{Conflicts of Interest}

The authors declare no conflicts of interest regarding the publication of this paper.

\section{References}

[1] Ge, J.B. and Xu, Y.J. (2013) Internal Medicine. 8th Edition, People's Medical Publishing House, 452.

[2] Wang, Y.-M., Gao, J., Xiong, Y.-M., et al. (2016) Analysis of the Causes of Lower Gastrointestinal Bleeding in 159 Elderly Patients. Military Medical Journal of South China, No. 9, 574-576.

[3] Qiu, Z.J. (2013) Clinical Analysis of 102 Cases of Lower Gastrointestinal Bleeding in 
the Elderly. China \& Foreign Medical Treatment, 32, 59.

[4] Sun, Y.C. (2015) Analysis of the Causes of 120 Cases of Gastrointestinal Bleeding. Contemporary Medicine Forum, No. 2, 181-182.

[5] He, X.-L. and Wang, W.-Y. (2013) Analysis of Etiology and Clinical Features of 266 Upper Gastrointestinal Bleeding Cases. Journal of Anhui Health Vocational \& Technical College, 12, 46-47.

[6] Liang, X., Bi, S., Yang, W., et al. (2013) Reprint of: Epidemiological Serosurvey of Hepatitis B in China-Declining HBV Prevalence due to Hepatitis B Vaccination. Vaccine, 31, J21-J28. https://doi.org/10.1016/j.vaccine.2013.08.012

[7] Guo, X.-L., Yao, S.-W., Cui, P.-L., et al. (2017) Single Center Analysis of Clinical Etiology of Lower Gastrointestinal Bleeding. World Journal of Gastrointestinal Endoscopy, No. 24, 2220-2223. https://doi.org/10.11569/wcjd.v25.i24.2220

[8] Hu, H.Y. (2013) The Cause Analysis of 590 Cases with Gastrointestinal Bleeding. Chinese Journal of Medicinal Guide, 15, 35-36.

[9] Hyung, S. (2014) What Can Be the Criteria of Outpatient-Based Endoscopic Resection for Colon Polyp? World Journal of Gastrointestinal Endoscopy, 6, 493.

https://doi.org/10.4253/wjge.v6.i10.493 